\title{
Synthesis of Bioactive Azetidinones of 4-Phenyl-1, 3-thiazole-2-amine
}

\author{
RAVINDRA KUMAR ${ }^{\mathrm{a}^{*}}$, ABHA SHUKLA ${ }^{\mathrm{b}}$ and D.S. TYAGI ${ }^{\mathrm{c}}$ \\ ${ }^{\text {a } D e p a r t m e n t ~ o f ~ C h e m i s t r y, ~ G u r u k u l ~ K a n g r i ~ V i s h w a v i d y a l a y a ~ H a r d w a r, ~ I n d i a ~}$ \\ ${ }^{\mathrm{b}}$ Department of Chemistry, K.G.M. Gurukul Kangri Vishwavidyalaya Hardwar, India \\ ${ }^{\mathrm{c}}$ Department of Chemistry, L.R. (P.G.) College, Sahibabad, India \\ ravi_tyagi18@rediffmail.com
}

Received 5 April 2013 / Accepted 8 May 2013

\begin{abstract}
Azetidinones have been synthesized by the cyclocondensation of chloroacetylchloride with Schiff base. The compounds have been characterized on the basis of analytical and spectral data. They have been screened of antibacterial activity against Bacillus subtilis, Staphylococcus aureus, E.coli and Salmonella typhi.
\end{abstract}

Keywords: Azetidinones, Schiff base, Antibacterial activity

\section{Introduction}

In recent years there has been considerable interest in the synthesis of substituted thiazolidinones derivatives due to their biological pharmacological activities ${ }^{1-3}$. Especially 4-thiazolidinones motifs are having many interesting activity profiles namely EOX-1 inhibitors ${ }^{4}$, inhibitors of the bacterial enzyme Mur - $\mathrm{B}^{5}$, non- nucleosides inhibitors of HfV-RT ${ }^{6}$ and anti histamine agents ${ }^{7}$. Thiazolidinones are the derivatives of thiazolidines which belong to an important group of heterocyclic compounds containing sulphur and nitrogen in a five member ring ${ }^{8-11}$. In search for new biodynamic potent molecule, it was thought worthwhile to incorporate some additional heterocyclic moieties in 4-thiazolidinones molecule and study their biological and pharmacological activity. The work plan given in the Scheme 1

\section{Experimental}

4-Phenyl-1,3-thiazole-2-amine was prepared accordingly to the literature method ${ }^{12}$. The aromatic benzaldehyde and substituted benzaldehyde were B.D.H. regents. Chemicals and solvents used were dried and purified by standard methods and moisture was excluded from the glass apparatus using $\mathrm{CaCl}_{2}$ drying tubes. 


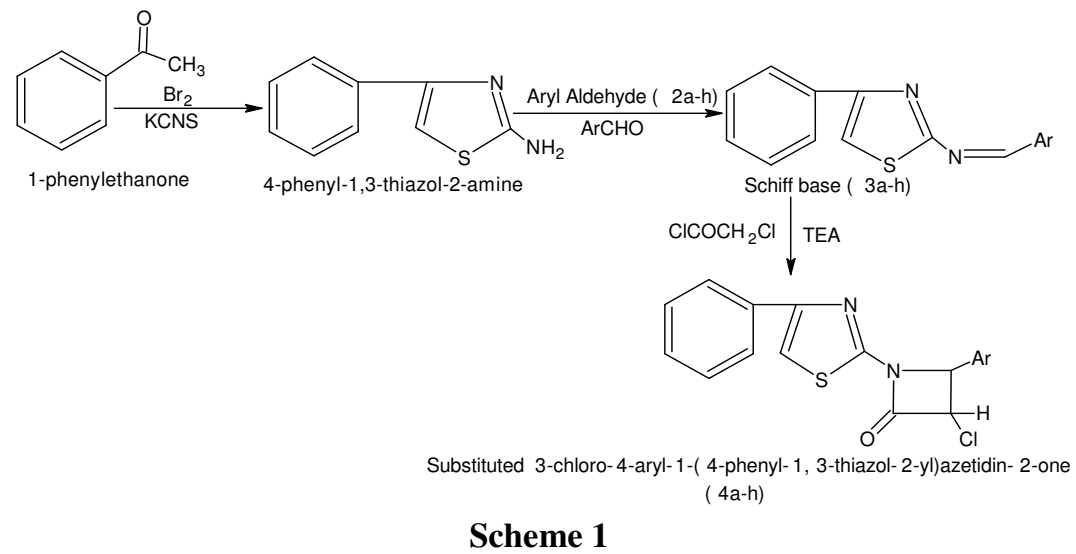

Where Ar is referred to as: (a) Phenyl (b) 4-methoxy phenyl (c) 4- hydroxy phenyl (d) 2hydroxy phenyl (e) 4-methyl phenyl (f) 3, 4- methylene dioxy phenyl (g) 4-hydroxy-3methoxy phenyl (h) 3.4- dimethoxy phenyl.

\section{Measurements}

The melting points were determined in open capillary tubes and are uncorrected. IR spectra were recorded on a Shimadzu FTIR-8400 instrument in $\mathrm{KBr}$ disc. ${ }^{1} \mathrm{H}$ NMR spectra were recorded on a Bruker AC-300 MHz FT NMR using TMS as internal standard, chemical shift are in $\delta$-ppm, mass spectra were recorded on a Jeol D-300 spectrometer. All the synthesized compounds gave satisfactory elementary analysis.

Antimicrobial activity of all the compounds were studied against gram positive bacteria (Bacillus Subtilis and Staphylococcus aureus) and gram negative bacteria (E. coli and Salmonella typhi) at a concentration of $50 \mu \mathrm{g} / \mathrm{mL}$ by agar cup method. Methanol system was used as control in the method ${ }^{13}$. Under similar conditions using penicillin and sulphanilamide as a standard, comparison carried at control experiment. The area of inhibition of zone is measured in percentage.

\section{Preparation of Schiff base (3a-h)}

To a solution of 4-phenyl-1, 3-thiazole-2-amine (1.75 g.0.01 mole) in $10 \mathrm{~mL}$ of ethanol $(10 \mathrm{~mL})$, substituted benzaldehyde $(1.06 \mathrm{~g}, 0.01 \mathrm{~mol})$ and $0.5 \mathrm{~mL}$ of piperidine as a catalyst was added. The reaction mixture was refluxed for 5-6 hours. Contents were cooled and poured on to crushed ice and thus the separated solid was isolated and crystallized from ethanol to give Schiff base (3a-h).Yield 60\%. The analytical data of all the compounds (3a-h) are given in Table 1 .

\section{Preparation of 2-azetidinones $(\mathbf{4 a}-\boldsymbol{h})$}

A mixture of Schiff base (3a-h) (0.002 m mole) and triethyl amine (TEA) (0.004 m mole) was dissolved in 1, 4- dioxane $(40 \mathrm{~mL})$ cool and stirred. Now, chloroacetyl chloride $(0.004 \mathrm{~m} \mathrm{~mol})(15 \mathrm{~mL})$ added slowly over a period of half an hour and contents further stirred for additional 3 hours and left at room temperature for 48 hours. The concentrated reaction mixture poured into ice cold water filters and then dried. Product was purified by column chromatography over silica gel using 30\% ethyl acetate: $70 \%$ benzene as eluant. Recrystallisation from $n$-hexane gave various derivatives of substituted 2-azetidinones in $65 \%$ yield. The analytical data of all the compounds $(\mathbf{4 a - h})$ are given in Table 2. 
Table 1. Analytical data of compounds 3a-h

\begin{tabular}{|c|c|c|c|c|c|c|c|c|}
\hline \multirow[b]{2}{*}{ Compd. } & \multirow{2}{*}{$\begin{array}{l}\text { Molecular } \\
\text { formula }\end{array}$} & \multirow{2}{*}{$\begin{array}{l}\text { Mol. Wt. } \\
\text { Found } \\
\text { (Calcd.) }\end{array}$} & \multirow{2}{*}{$\begin{array}{l}\text { Yield } \\
\%\end{array}$} & \multirow[b]{2}{*}{ M.P.C } & \multicolumn{4}{|c|}{ Chemical analysis \% Found (Calcd.) } \\
\hline & & & & & $\mathrm{C}$ & $\mathrm{H}$ & $\mathrm{N}$ & $S$ \\
\hline $3 \mathbf{a}$ & $\mathrm{C}_{16} \mathrm{H}_{12} \mathrm{~N}_{2} \mathrm{~S}$ & $\begin{array}{c}248 \\
(264.3)\end{array}$ & 67 & 155 & $\begin{array}{c}72.49 \\
(72.70)\end{array}$ & $\begin{array}{c}4.42 \\
(4.58)\end{array}$ & $\begin{array}{c}10.47 \\
(10.60)\end{array}$ & $\begin{array}{c}11.91 \\
(12.13)\end{array}$ \\
\hline $3 \mathbf{b}$ & $\mathrm{C}_{17} \mathrm{H}_{14} \mathrm{~N}_{2} \mathrm{O}_{2} \mathrm{~S}$ & $\begin{array}{c}282 \\
(294.3)\end{array}$ & 63 & 153 & $\begin{array}{c}69.18 \\
(69.36)\end{array}$ & $\begin{array}{c}4.68 \\
(4.79)\end{array}$ & $\begin{array}{c}9.46 \\
(9.52)\end{array}$ & $\begin{array}{c}10.73 \\
(10.89)\end{array}$ \\
\hline $3 \mathbf{c}$ & $\mathrm{C}_{16} \mathrm{H}_{12} \mathrm{~N}_{2} \mathrm{OS}$ & $\begin{array}{c}264 \\
(280.3)\end{array}$ & 57 & 146 & $\begin{array}{c}68.32 \\
(68.55)\end{array}$ & $\begin{array}{c}4.13 \\
(4.31)\end{array}$ & $\begin{array}{c}9.84 \\
(9.99)\end{array}$ & $\begin{array}{c}11.23 \\
(11.44)\end{array}$ \\
\hline 3d & $\mathrm{C}_{16} \mathrm{H}_{12} \mathrm{~N}_{2} \mathrm{OS}$ & $\begin{array}{c}264 \\
(280.3)\end{array}$ & 63 & 150 & $\begin{array}{c}68.32 \\
(68.55)\end{array}$ & $\begin{array}{c}4.10 \\
(4.31)\end{array}$ & $\begin{array}{c}9.84 \\
(9.99)\end{array}$ & $\begin{array}{c}11.23 \\
(11.44)\end{array}$ \\
\hline $3 \mathbf{e}$ & $\mathrm{C}_{17} \mathrm{H}_{14} \mathrm{~N}_{2} \mathrm{~S}$ & $\begin{array}{c}254 \\
(278.4)\end{array}$ & 65 & 140 & $\begin{array}{c}73.14 \\
(73.35)\end{array}$ & $\begin{array}{c}4.90 \\
(5.07)\end{array}$ & $\begin{array}{c}9.86 \\
(10.06)\end{array}$ & $\begin{array}{c}11.35 \\
(11.52)\end{array}$ \\
\hline $3 f$ & $\mathrm{C}_{17} \mathrm{H}_{12} \mathrm{~N}_{2} \mathrm{O}_{2} \mathrm{~S}$ & $\begin{array}{c}292 \\
(308.4)\end{array}$ & 58 & 157 & $\begin{array}{c}65.94 \\
(66.22)\end{array}$ & $\begin{array}{c}3.71 \\
(3.92)\end{array}$ & $\begin{array}{c}8.90 \\
(9.08)\end{array}$ & $\begin{array}{c}10.21 \\
(10.38)\end{array}$ \\
\hline $3 g$ & $\mathrm{C}_{17} \mathrm{H}_{14} \mathrm{~N}_{2} \mathrm{O}_{2} \mathrm{~S}$ & $\begin{array}{c}294 \\
(310.4)\end{array}$ & 54 & 160 & $\begin{array}{c}65.43 \\
(65.79)\end{array}$ & $\begin{array}{c}4.35 \\
(4.55)\end{array}$ & $\begin{array}{c}8.88 \\
(9.03)\end{array}$ & $\begin{array}{c}10.16 \\
(10.33)\end{array}$ \\
\hline $3 \mathbf{h}$ & $\mathrm{C}_{18} \mathrm{H}_{16} \mathrm{~N}_{2} \mathrm{O}_{2} \mathrm{~S}$ & $\begin{array}{c}307 \\
(324.4) \\
\end{array}$ & 53 & 154 & $\begin{array}{r}66.45 \\
(66.64) \\
\end{array}$ & $\begin{array}{c}4.79 \\
(4.97) \\
\end{array}$ & $\begin{array}{r}8.44 \\
(8.64) \\
\end{array}$ & $\begin{array}{c}9.52 \\
(9.88) \\
\end{array}$ \\
\hline
\end{tabular}

Table 2. Analytical and spectral data of compounds (4a-h)

\begin{tabular}{|c|c|c|c|c|c|c|c|c|c|c|c|c|c|}
\hline \multirow{3}{*}{\multicolumn{2}{|c|}{ 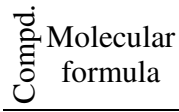 }} & \multirow{3}{*}{$\frac{\dot{z}}{\dot{0}}$} & \multirow{3}{*}{$\frac{\partial}{2} \stackrel{0}{\Sigma}$} & \multicolumn{9}{|c|}{ Analysis } & \multirow{3}{*}{ PMR ( $\delta$ PPM) } \\
\hline & & & & \multirow{2}{*}{\multicolumn{3}{|c|}{$\frac{\% \mathrm{C}}{\mathrm{Cald}}$}} & \multicolumn{2}{|c|}{$\% \mathrm{H}$} & \multicolumn{2}{|c|}{$\% \mathrm{~N}$} & \multicolumn{2}{|c|}{$\% \mathrm{~S}$} & \\
\hline & & & & & & & Cald. & Foun & Cald. & Found & d Cald.I & Found & \\
\hline $4 \mathbf{a}$ & $\begin{array}{c}\mathrm{C}_{18} \mathrm{H}_{13} \\
\mathrm{~N}_{2} \mathrm{SOCl}\end{array}$ & 340.5 & 651 & 806 & 63.44 & 463.30 & 3.82 & 3.70 & 8.22 & 8.10 & 9.4 & 9.20 & $\begin{array}{l}\text { 9-2 } 1 \mathrm{Hd} \mathrm{C}_{3}, \mathrm{H} \text { 9-2-7-8 } \\
\left(10 \mathrm{H} \mathrm{mtd} \text { aromatic } \mathrm{C}_{4} \mathrm{H}\right.\end{array}$ \\
\hline & $\begin{array}{c}\mathrm{C}_{19} \mathrm{H}_{15} \\
\mathrm{~N}_{2} \mathrm{SO}_{2} \mathrm{Cl}\end{array}$ & 370. & 601 & & 61.54 & +61.40 & 4.05 & 3.97 & 7.56 & 7.40 & 8.64 & 8.50 & $\begin{array}{c}\text { 8-2-7.8 }(9 \mathrm{H} \mathrm{mtd} \\
\text { aromatic \& } \mathrm{C}_{4} \mathrm{H}, 9.2 \\
(\mathrm{Hd} . \mathrm{C} 3 \mathrm{H}) 2.1\left(3 \mathrm{H}_{3} \mathrm{CH}_{3}\right)\end{array}$ \\
\hline & $\begin{array}{c}\mathrm{C}_{18} \mathrm{H}_{13} \\
\mathrm{~N}_{2} \mathrm{SO}_{2} \mathrm{Cl}\end{array}$ & 356.5 & 551 & & 60.59 & 60.42 & 3.65 & 3.50 & 7.85 & 7.70 & 8.98 & 8.80 & $\begin{array}{c}\text { 8-2-9.8 (9 H mtd } \\
\text { aromatic \& } \mathrm{C}_{4} \mathrm{H}, 9.2 \\
\left(\mathrm{Hd}_{3} \mathrm{C}_{3} \mathrm{H} 3.9(\mathrm{H} \mathrm{S} \mathrm{OH})\right.\end{array}$ \\
\hline & $\begin{array}{c}\mathrm{C}_{18} \mathrm{H}_{13} \\
\mathrm{~N}_{2} \mathrm{SO}_{2} \mathrm{Cl}\end{array}$ & 356.5 & 601 & & 60.59 & 60.42 & 3.65 & 3.47 & 7.85 & 7.70 & 8.98 & 8.80 & $\begin{array}{c}\text { 8-2-9.8 }(9 \mathrm{H} \mathrm{mtd} \\
\text { aromatic \& } \mathrm{C}_{4} \mathrm{H}, 9.2 \\
\left(\mathrm{Hd} . \mathrm{C}_{3} \mathrm{H} 3.9(\mathrm{H} \mathrm{S} \mathrm{OH})\right.\end{array}$ \\
\hline & $\begin{array}{c}\mathrm{C}_{19} \mathrm{H}_{15} \\
\mathrm{~N}_{2} \mathrm{SOCl}\end{array}$ & 354.5 & 651 & & 64.32 & 264.18 & 4.23 & 4.10 & 7.9 & 7.70 & 9.03 & 8.88 & $\begin{array}{c}\text { 8-2-9.8 }(9 \mathrm{H} \mathrm{mtd} \\
\text { aromatic \& } \mathrm{C}_{4} \mathrm{H}, 9.2 \\
\left(\mathrm{Hd}_{3} \mathrm{C}_{3} \mathrm{H} 3.9(\mathrm{H} \mathrm{S} \mathrm{OH})\right.\end{array}$ \\
\hline & $\begin{array}{c}\mathrm{C}_{19} \mathrm{H}_{13} \\
\mathrm{~N}_{2} \mathrm{SO}_{3} \mathrm{Cl}\end{array}$ & 384.5 & 551 & 50 & 59.3 & 59.10 & 3.38 & 3.20 & 7.28 & 7.10 & 8.32 & 8.15 & $\begin{array}{c}\text { 8-2-7.8 }(8 \mathrm{H} \mathrm{mtd} \\
\text { aromatic \& } \mathrm{C}_{4} \mathrm{H}, 9.2 \\
\left(\mathrm{Hd} . \mathrm{C}_{3} \mathrm{H} 3.9(\mathrm{H} \mathrm{S} \mathrm{OH})\right.\end{array}$ \\
\hline & $\begin{array}{c}\mathrm{C}_{19} \mathrm{H}_{15} \\
\mathrm{~N}_{2} \mathrm{SO}_{3} \mathrm{Cl}\end{array}$ & 386.5 & 521 & & 58.99 & 958.80 & 3.88 & 3.72 & 7.24 & . 7.10 & 8.28 & 8.12 & $\begin{array}{c}\text { 8-2-9.8 (8 H mtd } \\
\text { aromatic \& } \mathrm{C}_{4} \mathrm{H}, 9.2 \\
\left(\mathrm{Hd}_{\mathrm{C}} \mathrm{C}_{3} \mathrm{H} 3.9(\mathrm{H} \mathrm{S} \mathrm{OH})\right.\end{array}$ \\
\hline 41 & $\begin{array}{c}\mathrm{C}_{20} \mathrm{H}_{17} \\
\mathrm{~N}_{2} \mathrm{SO}_{3} \mathrm{Cl}\end{array}$ & 400 & 50 & & 59.93 & 359.80 & 4.24 & 4.10 & 6.99 & 6.80 & 7.99 & 7.85 & $\begin{array}{c}\text { 8-2-9.8 }(8 \mathrm{H} \mathrm{mtd} \\
\text { aromatic \& } \mathrm{C}_{4} \mathrm{H}, 9.2 \\
\left(\mathrm{Hd} . \mathrm{C}_{3} \mathrm{H} 3.9(\mathrm{H} \mathrm{S} \mathrm{OH})\right.\end{array}$ \\
\hline
\end{tabular}




\section{Results and Discussion}

4-Phenyl-1,3-thiazole-2-amine ${ }^{14}$ solution in ethanol, on reaction with aromatic aldehydes in presence of piperidine gave Schiff bases $(\mathbf{3 a}-\mathbf{h})$, which were characterized by elemental analysis IR and NMR spectral studies ${ }^{15-18}$. The IR spectra of the Schiff base show the prominent bands of $1630-20 \mathrm{~cm}^{-1}$ for the azomethine group.

The Schiff base on cyclocondensation reaction with chloroacetyl chloride ${ }^{19-21}$ gave substituted 2-azetidinones (4a-h). The structure of these were established on the basis of chemical analysis, IR (cyclic $>\mathrm{C}=\mathrm{O}$ group, $1680 \mathrm{~cm}^{-1}$ ) and NMR signals for different kinds of protons at their respective positions.

Antibacterial activity

The antibacterial activities of the series (4a-h) have been carried out against some strain of bacteria. The result (Table 3) shows that the prepared compounds are toxic against the bacteria. 4b, 4c and 4d, were found more active against the above microbes. The comparison of the antibacterial activity of these compounds with penicillin shows that these compounds have almost similar activity.

Table 3. Antibacterial activity of the compounds (4a-h)

\begin{tabular}{ccccc}
\hline \multirow{2}{*}{ Compound } & \multicolumn{4}{c}{ \% of Zone of inhibition } \\
\cline { 2 - 5 } & \multicolumn{3}{c}{ gram +ve } & \multicolumn{3}{c}{ gram -ve } \\
\cline { 2 - 5 } & Bacillus subtillis & Staphylococcus aureus & E.coli & Salmonella typhi \\
\hline $\mathbf{4 a}$ & 56 & 65 & 42 & 66 \\
$\mathbf{4 b}$ & 46 & 74 & 54 & 71 \\
$\mathbf{4 c}$ & 73 & 83 & 78 & 63 \\
$\mathbf{4 d}$ & 83 & 68 & 68 & 78 \\
$\mathbf{4 e}$ & 44 & 65 & 46 & 73 \\
$\mathbf{4 f}$ & 68 & 61 & 63 & 50 \\
$\mathbf{4 g}$ & 71 & 54 & 68 & 60 \\
$\mathbf{4 h}$ & 81 & 71 & 78 & 73 \\
Penicillin & 83 & 63 & 73 & 73 \\
\hline
\end{tabular}

\section{Acknowledgement}

The authors are thankful to Head, Department of Chemistry, Gurukul Kangri University, Haridwar and Head, department of chemistry, L.R.P.G.College, Sahibabad, Ghaziabad for encouraging in literary survey and providing the research facilities.

\section{References}

1. Reifschulider W, Bisabari B E, Dripps J E and Bouron J B, US Patent 5,075, 1991, 293; Chem/ Abstr., 1991, 116, 29249 f.

2. Deepak Kumar, Bux F B and Arun Singh, Rasayan J Chem., 2010, 3(3), 497-502.

3. Jubie S, Gowramma B, Nitin K Muthal, Kalirajan R, Gomathi S and Elango K, Int J Chem Res., 2009, 1(2), 153-157.

4. Look G C, Schullek J R, Holmes C p, Chinu J P, Gordon E M and Gallop M A, Bioorg Med Chem Lett., 1996, 6(6), 707-712.

5. Anders C J, Bronson J J, D'Andrla S V, Deshpande S M, Falk P J, Grant Young K A, Harte W E, HO H, Misco P F, Robertson J G, Stock D, Sun Y and Walsh A W, Bioorg Med Chem Lett., 2001, 715. 
6. Barreca M L, Chimirri A, Luca L D, Monforte A, Monforte P, Rao A, Zappala M, Balzarrini J, De Clareq E, Pannecouque C and Wifvrouw M, Bioorg Med Chem Lett., 2001, 1793.

7. Diurno M V, Mazzoni O, Eugenio P, Calignano A, Giordano F and Bolognese A, $J$ Med Chem., 1992, 35(15), 2910-2912.

8. Palaska E, Sachin G, Kelicen P, Durlu N T and Altinok G, Farmaco, 2002, 57, 101-107.

9. Dundar Y, Cakir B, Kupel E, Sahin M F and Noyanalpan N, Turk J Chem., 2007, 31, 301.

10. Amir M and Kumar S, Acta Pharm., 2007, 57, 31-47.

11. El-Sayed R, Indian J Chem., 2006, 45B, 738-746.

12. Tripathy H and Mahaputra G N, J Indian Chem Soc., 1975, 52, 168.

13. Barry A L, The Antimicrobial Susceptibility Test: Principle and Practices, Edited by Illusles and Febiger (Philadelphia, USA) 1976, 180; Biol Abstr., 1976, 64, 25183.

14. Kemp W, Organic Spectroscopy, $2^{\text {nd }}$ Edition Basingtake, Macmillan USA.

15. Akitt J W, NMR and Chemistry, $2^{\text {nd }}$ Ed., Chapmam \& Hall, 1983.

16. Brcitmair E and Voelter W, ${ }^{13} \mathrm{C}$ NMR Spectroscopy; High resolution methods and applications in organic chemistry and biochemistry, VCH- publication, Germany, 1990.

17. Wehrli F W, Marchand A P and Wlhrli S, Interpretation of Carbon-13 NMR Spectra $2^{\text {nd }}$ Ed., John Wiley \& Sons Ltd; Tiptree, Essex, UK, 1988, pp. 164-184.

18. Levy G C, Lichter R L and Nelson G L, Carbon-13 Nuclear Magnetic Resonance Spectroscopy, $2^{\text {nd }}$ Ed., Wiley New York, 1980a.

19. Srivastava V K, Singh Shridha, Kumar P and Shanker S K, Indian J Chem., 1986, 25B, 769-771.

20. Rawat T R and Srivastava S D, Indian J Chem., 1998, 37B, 91.

21. Deshmukh M B and Deshmukh D S, J Indian Chem Soc., 1995, 72, 847. 